\title{
GENERACIÓN FOTOSENSIBILIZADA DE OXÍGENO SINGLETE
}

\section{PHOTOSENSIBILITY GENERATION OF SINGLET OXYGEN}

\author{
David García Fresnadillo ${ }^{1}$
}

\begin{abstract}
RESUMEN
La presente comunicación versa sobre la producción fotosensibilizada de oxígeno singlete. A lo largo de la misma se pretenden transmitir las siguientes ideas básicas: ¿Qué es el oxígeno singlete?, ¿Cómo se puede generar esta especie química?, prestando especial atención al método de la fotosensibilización. Una vez producido el oxígeno singlete, ¿cómo se puede evaluar y cuantificar su producción? y finalmente, ¿cuál es la reactividad del oxígeno singlete?, por lo tanto, ¿cuáles son los potenciales ámbitos de aplicación de esta especie química?.
\end{abstract}

Palabras claves.- Fotosensibilizada, Oxigeno singlete.

\begin{abstract}
The present communication deals with the production of singlet oxygen photosensibility along the same it is intended to convey the following basic ideas: ¿What is the singlet oxygen? ¿How can you generate this chemical species?, paying special attention to the method of photosensitisation. Once produced the singlet oxygen, ¿how can you evaluate and quantify its production? and finally, ¿what is the reactivity of the singlet oxygen?, therefore, ¿what are the potential areas of application of this chemical species?.
\end{abstract}

Keywords.- Photosensibility, Singlete oxygen.

\section{INTRODUCCION}

\section{OXÍGENO SINGLETE}

El oxígeno molecular o dioxígeno es una especie química ubicua cuyas propiedades físico-químicas derivan de su configuración electrónica. Considerando el diagrama de orbitales moleculares para el dioxígeno en estado fundamental, es decir, con su configuración electrónica de mínima energía, se tienen dos electrones desapareados o de espines paralelos en un nivel $\pi^{*}$, por lo que se trata de un estado de multiplicidad triplete, denominado ${ }^{3} \sum_{g}^{-}$según la notación de los espectroscopistas (Figura 1). Por otro lado, para las dos siguientes configuraciones electrónicas en orden de energía creciente, se tienen dos configuraciones singlete con electrones apareados o de espines contrarios en el nivel $\pi^{*}$, que dan lugar a dos estados excitados de mayor energía, denominados ${ }^{1} \Delta_{\mathrm{g}}$ y ${ }^{1} \sum_{\mathrm{g}}{ }^{+}$según la notación de los espectroscopistas.

Dichos estados excitados o activados, a diferencia del estado fundamental, presentan configuraciones electrónicas que tienen una duración limitada en el tiempo, por lo que ambos tienden a regresar a la configuración de mínima energía del estado fundamental pasado un corto lapso de tiempo.

Este periodo se conoce como tiempo de vida y es característico de cada estado y del medio que circunda a las moléculas.

Atendiendo a la diferencia de energía entre los dos estados excitados singlete del dioxígeno y el estado fundamental triplete, así como a su duración, el estado $\mathrm{O}_{2}\left({ }^{1} \sum_{\mathrm{g}}{ }^{+}\right)$posee un exceso de energía de $156.9 \mathrm{~kJ} \mathrm{~mol}^{-1}$ respecto del estado fundamental y un tiempo de vida de 7-12 s en fase gaseosa, $\mathrm{y} \approx 1$ ps en disolución.

${ }^{T}$ Prof. Dr. Laboratorio de Fotoquímica Aplicada, Departamento de Química Orgánica, Facultad de Ciencias Químicas, Universidad Complutense de Madrid, España. 


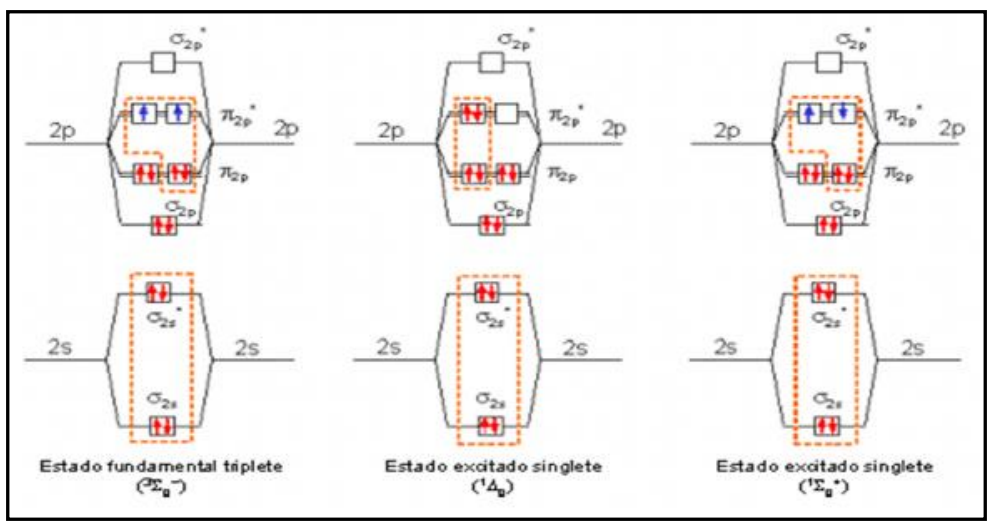

Fig. 1 Diagrama de orbitales moleculares para el nivel $n=2$ de la molécula de oxígeno en estado fundamental y para sus dos estados excitados de menor energía..

Por su parte, el estado $\mathrm{O}_{2}\left({ }^{1} \Delta_{\mathrm{g}}\right)$ tiene un exceso de energía de $94.2 \mathrm{~kJ} \mathrm{~mol}^{-1}$ sobre el estado fundamental y una duración del orden de minutos en medio gaseoso, o en el intervalo de $4 \mu$ s a 60 ms en fase líquida, dependiendo del disolvente. El estado $\mathrm{O}_{2}\left({ }^{1} \sum_{\mathrm{g}}{ }^{+}\right)$, por su menor tiempo de vida, tiende a desactivarse rápidamente para alcanzar la configuración del estado $\mathrm{O}_{2}\left({ }^{1} \Delta_{\mathrm{g}}\right)$ por lo que, en general, se denomina habitualmente oxígeno singlete o ${ }^{1} \mathrm{O}_{2}$ al estado excitado $\mathrm{O}_{2}\left({ }^{1} \Delta_{\mathrm{g}}\right)$ de la molécula de oxígeno (Figura 2 ). Su exceso de energía respecto al estado fundamental hace que esta especie excitada presente una importante reactividad química durante su tiempo de vida.

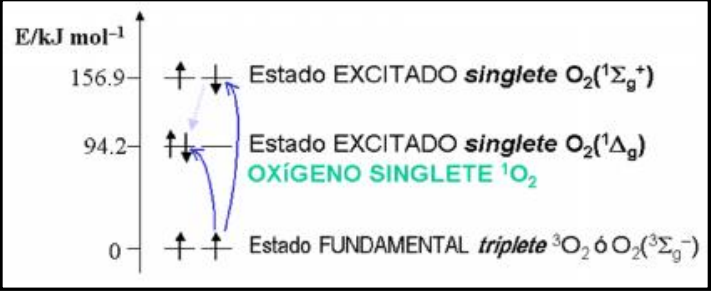

Fig. 2 Diagrama de estados de la molécula de oxígeno en su estado fundamental y en sus dos estados excitados singlete.

\section{GENERACIÓN DE OXÍGENO SINGLETE}

Para generar oxígeno singlete se puede acudir a métodos químicos térmicos o bien a métodos fotoquímicos (Figura 3).

Entre los métodos químicos térmicos destacan (a) la reacción del peróxido de hidrógeno o agua oxigenada con hipoclorito sódico o potásico; (b) la descomposición térmica de fosfitozónidos generados a partir de trialquil o triarilfosfitos y ozono a baja temperatura; y (c) la descomposición térmica de endoperóxidos de compuestos aromáticos. Estos métodos son útiles en el laboratorio para la generación de oxígeno singlete a pequeña escala, pero nunca para la producción de oxígeno singlete en grandes cantidades, ya que hacen uso de reactivos altamente corrosivos (a) o de reactivos químicos sofisticados de difícil preparación y alto costo (b) y (c). La fotosensibilización, por su parte, constituye el método de elección para la preparación de oxígeno singlete, por su asequibilidad ya sea a pequeña o a gran escala, puesto que hace uso de reactivos económicos (colorantes de fácil acceso por su escaso coste así como luz ultravioleta o visible) y no requiere montajes experimentales de especial complejidad.

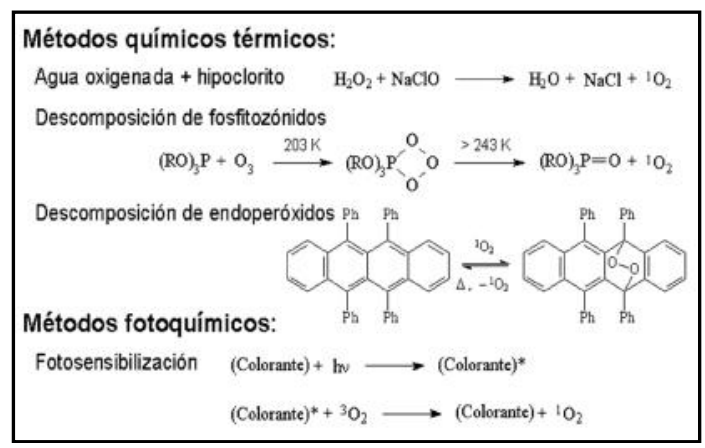

Fig. 3 Métodos químicos térmicos y fotoquímicos para la generación de oxígeno singlete. 
El proceso de fotosensibilización se produce al generar, por absorción de luz de longitud de onda adecuada, el estado excitado de las moléculas de un colorante determinado llamado sensibilizador.

El exceso de energía de excitación de las moléculas de dicho colorante se transfiere al oxígeno mediante choque con las moléculas de dioxígeno presentes en el medio, durante el tiempo de vida del estado excitado del sensibilizador, resultando así la formación de oxígeno singlete (Figura 3). Visto a nivel molecular, el mecanismo de la fotosensibilización se produce típicamente desde el estado excitado triplete del sensibilizador, el cual se desactiva por colisión con una molécula de dioxígeno a través de un proceso de transferencia de energía por intercambio electrónico (Figura 4). El resultado de dicha colisión es la producción de una molécula de oxígeno singlete y la recuperación del sensibilizador en estado fundamental, por lo que el proceso de fotosensibilización se considera como un proceso fotocatalítico con consumo exclusivo de fotones y dioxígeno.

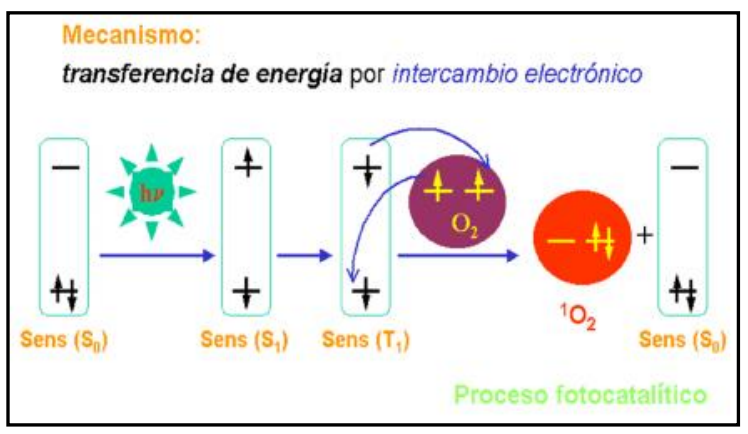

Fig.4 Mecanismo del proceso de fotosensibilización para la fotogeneración de oxígeno singlete. Sens = sensibilizador; $S_{0}=$ estado fundamental del sensibilizador; $S_{1}$ y $T_{1}$ son los estados excitados singlete $y$ triplete del sensibilizador, respectivamente; $h v$ representa el proceso de absorción de un fotón de luz UV-Vis por parte del sensibilizador.

El balance energético del proceso de sensibilización se puede determinar teniendo en cuenta su diagrama de Jablonski o de estados energéticos involucrados (Figura 5). Según él, tras la absorción de un fotón (hv) por parte del sensibilizador en estado fundamental $\left(\mathrm{S}_{0}\right)$, se genera el estado excitado singlete $\left(\mathrm{S}_{1}\right)$. El exceso de energía vibrorrotacional del mismo se disipa a través de un proceso de relajación vibracional $(\mathrm{RV})$, normalmente una vibración molecular que ocurre en menos de 1 ps. Desde $S_{1}$ ocurre un proceso de cruce intersistemas (CIS) que requiere la inversión del espín del electrón excitado, este proceso es más lento y requiere alrededor de $1 \mathrm{~ns}$ para suceder. De este modo, tras la necesaria relajación vibracional se alcanza el nivel triplete excitado $\left(\mathrm{T}_{1}\right)$ más bajo del sensibilizador, a partir del cual puede ocurrir el proceso de transferencia de energía por intercambio electrónico si hay moléculas de oxígeno presentes en el medio. Este proceso es competitivo con la relajación del sensibilizador excitado al estado fundamental (proceso no radiante) y con la emisión de un fotón de luminiscencia hv" (proceso radiante). Una vez ocurrida la transferencia de energía se alcanza rápidamente el estado excitado singlete ${ }^{1} \Delta_{\mathrm{g}}$ del dioxígeno, el cual puede reaccionar durante su tiempo de vida con alguna molécula reactiva presente en el medio, o bien desactivarse al estado fundamental del dioxígeno a través de un proceso radiante (emisión de un fotón de fosforescencia a $1270 \mathrm{~nm}$ ) o no radiante.

Entre los requisitos que debe poseer todo sensibilizador para que este proceso suceda de forma eficiente se encuentran los siguientes:

- Intensa absorción de luz UV-Vis (preferentemente en la región visible) con coeficientes de absorción molar ( ) superiores a $10^{4} \mathrm{M}^{-1} \mathrm{~cm}^{-1}$.

- Poseer un elevado rendimiento cuántico de cruce intersistemas $\left({ }_{\mathrm{CIS}}\right)$, próximo a la unidad, así como una energía del estado triplete $\left(\mathrm{T}_{1}\right)$ superior a la del oxígeno singlete.

- Largos tiempos de vida del estado excitado $\left(\mathrm{T}_{1}\right)$, en el orden del $\mu$ s o superior.

- Alto rendimiento cuántico de producción de oxígeno singlete $\left({ }^{\Phi} \Delta\right)$ en distintos medios.

- Buena estabilidad térmica y fotoquímica.

- Capacidad de ser inmovilizados en soportes poliméricos de modo asequible. 


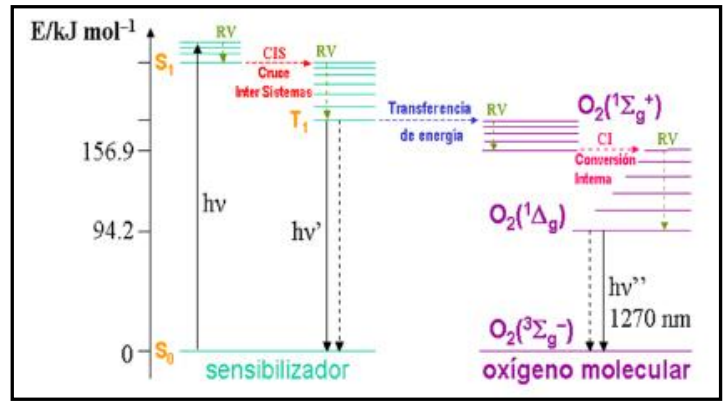

Fig. 5 Diagrama de Jablonski para el proceso de sensibilización del oxígeno singlete. $R V=$ proceso de relajación vibracional; $C I S=$ proceso de cruce intersistemas de los estados $S_{1}$ a $T_{1}$ del sensibilizador; $h v^{\prime}=$ emisión de un fotón de luminiscencia del sensibilizador; $\mathrm{CI}=$ proceso de conversión interna de los estados $O_{2}\left({ }^{1} \sum_{g}^{+}\right)$ a $\mathrm{O}_{2}\left({ }^{l} \Delta_{g}\right)$ del oxígeno; $h v^{\prime \prime}=$ emisión de un fotón de fosforescencia del oxígeno singlete. Las líneas de trazos representan procesos fotofísicos no radiantes mientras que las continuas representan procesos radiantes.

Los sensibilizadores más comúnmente utilizados para la generación de oxígeno singlete son la fenalenona $(\phi \Delta=1)$, el rosa de bengala $(\phi \Delta=$ $0.75)$, el azul de metileno $(\phi \Delta=0.5)$, los complejos de rutenio(II) con ligandos poliazaheterocíclicos quelatantes del tipo 2,2'-bipiridina o 1,10fenantrolina y sus derivados $(\phi \Delta=0.2-1)$ así como los derivados de porfirinas y ftalocianinas, y sus complejos metálicos (Figura 6).

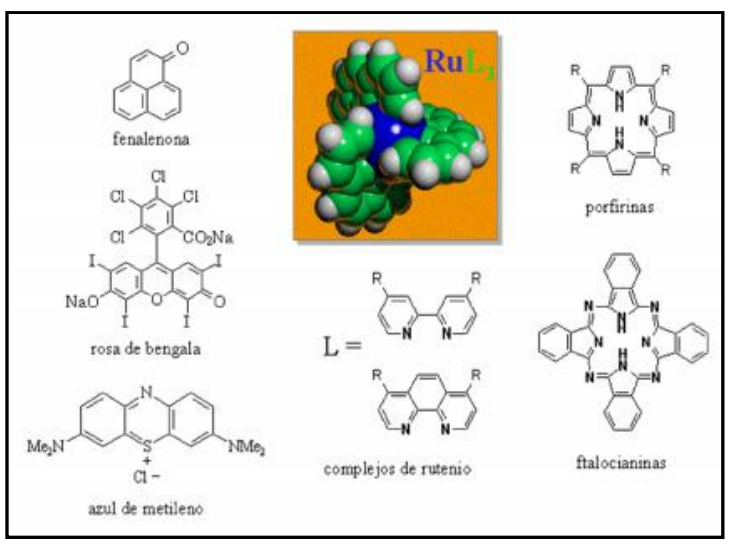

Fig. 6 Sensibilizadores típicamente usados en la producción de oxígeno singlete.

\section{EVALUACIÓN DE LA PRODUCCIÓN DE OXÍGENO SINGLETE}

Al cuantificar la fotosensibilización de oxígeno singlete es necesario atender, por un lado, a la eficiencia del proceso de desactivación del estado excitado del sensibilizador por dioxígeno y, por otro, a la propia eficiencia de generación de oxígeno singlete.

En cuanto a la eficiencia del proceso de desactivación del estado excitado del sensibilizador, ésta viene determinada por la constante de velocidad de desactivación colisional bimolecular $\left(\mathrm{k}_{\mathrm{q}}\right)$ que normalmente está controlada por la propia difusión de las moléculas en el medio donde tiene lugar la fotorreacción, alcanzando valores próximos a $10^{10} \mathrm{M}^{-1} \mathrm{~s}^{-1}$ en fase líquida y siendo uno o dos órdenes de magnitud menor en medios sólidos.

Dicha constante de velocidad puede estimarse fácilmente a partir de medidas experimentales de emisión del sensibilizador en estado estacionario o con resolución temporal y aplicando la ecuación de Stern-Volmer:

$$
\mathrm{I}_{0} / \mathrm{I}=\tau_{0} / \tau=1+\mathrm{k}_{\mathrm{q}} \tau_{0}\left[\mathrm{O}_{2}\right]
$$

donde $\mathrm{I}_{0}$ e I son, respectivamente, las intensidades de luz de emisión del sensibilizador determinadas en ausencia y en presencia del desactivador de los estados excitados, en este caso el dioxígeno; $\tau_{0}$ y $\tau$ son los tiempos de vida de emisión del sensibilizador determinados en ausencia y en presencia del desactivador, respectivamente y $\left[\mathrm{O}_{2}\right]$ es la concentración del desactivador, en nuestro caso el oxígeno molecular, en el medio.

A partir de estos parámetros experimentales también puede determinarse la eficiencia del proceso de desactivación del estado excitado del sensibilizador por dioxígeno $\left(\mathrm{Po}_{2}{ }^{\mathrm{T}}\right)$, que viene dada por la siguiente expresión:

$$
\mathrm{Po}_{2}{ }^{\mathrm{T}}=1-\tau / \tau_{0}=\tau \mathrm{k}_{\mathrm{q}}\left[\mathrm{O}_{2}\right]
$$

y que depende del tipo de sensibilizador, la concentración de oxígeno, así como el tipo de medio donde ocurre el proceso. 
En lo que concierne a la eficiencia de producción de oxígeno singlete, es importante la eficiencia de generación de oxígeno singlete a partir de todos los eventos de desactivación del estado excitado del sensibilizador $\left(\mathrm{f}_{\Delta}^{\mathrm{T}}\right)$, que depende principalmente del medio donde ocurre la sensibilización y de las interacciones de transferencia de carga entre el sensibilizador y el dioxígeno.

Finalmente, el parámetro primordial es el rendimiento cuántico de producción de oxígeno singlete ( ), el cual, se relaciona con los anteriores de la siguiente manera:
A la hora de caracterizar el oxígeno singlete fotogenerado, es decir, determinar su tiempo de vida y el rendimiento cuántico de producción de oxígeno singlete, se aprovechan dos de sus propiedades físico-químicas más importantes: su emisión de luz en la región del infrarrojo cercano centrada a $1270 \mathrm{~nm}$ (Figura 7) y su carácter electrófilo (Figura 8) lo que le hace ser bastante reactivo hacia otras moléculas ricas en densidad electrónica como aquellas con enlaces múltiples carbono-carbono y con grupos funcionales con heteroátomos electronegativos (nitrógeno, oxígeno y azufre) que funcionan como moléculas "atrapadoras" de oxígeno singlete.

$$
\phi_{\Delta}=\phi_{\mathrm{CIS}} \mathrm{Po}_{2}{ }^{\mathrm{T}} \mathrm{f}_{\Delta}^{\mathrm{T}}
$$

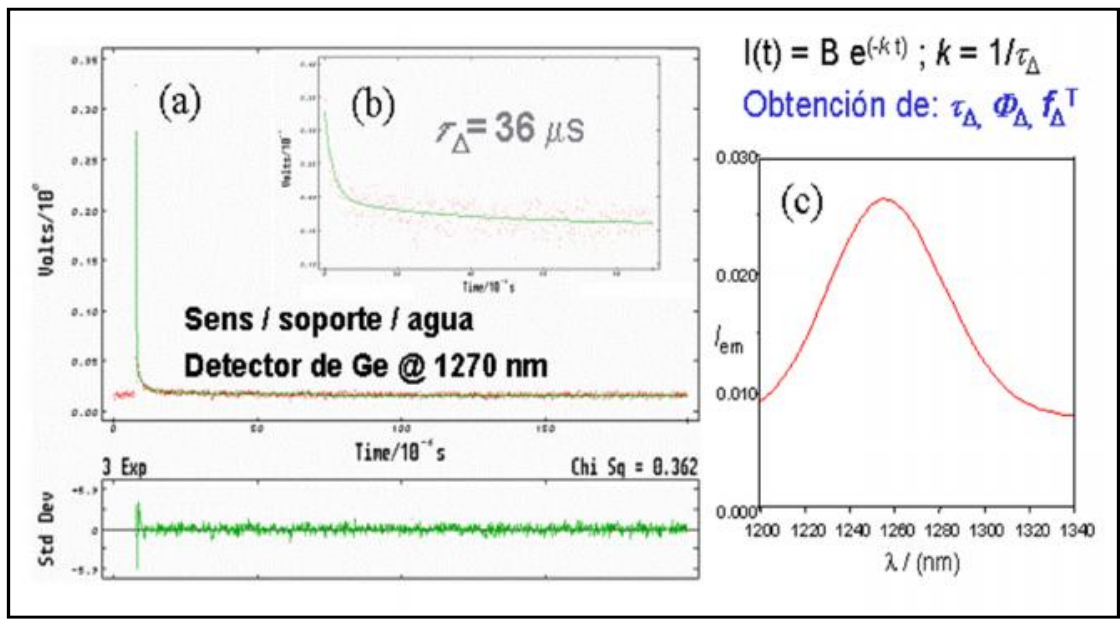

Fig. 7 (a) Cinética de la emisión del oxígeno singlete a $1270 \mathrm{~nm}$, producida por un sensibilizador inmovilizado en un soporte polimérico sumergido en agua y obtenida con un equipo de fotólisis de destello láser. (b) Ampliación del decaimiento de la señal de emisión del oxígeno singlete medida con un detector de Ge, determinándose un tiempo de vida de $36 \quad s$ mediante ajuste de los puntos experimentales a una función exponencial. El factor preexponencial $(B)$ es proporcional a la cantidad de oxígeno singlete fotogenerado. (c) Espectro de emisión del oxígeno singlete en la región del infrarrojo cercano. 
(a)

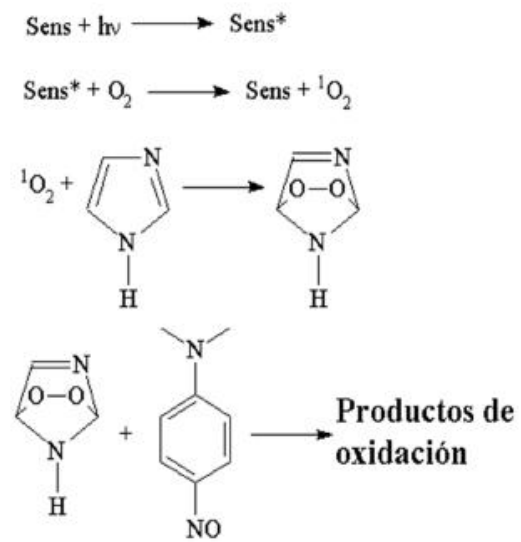

(b)

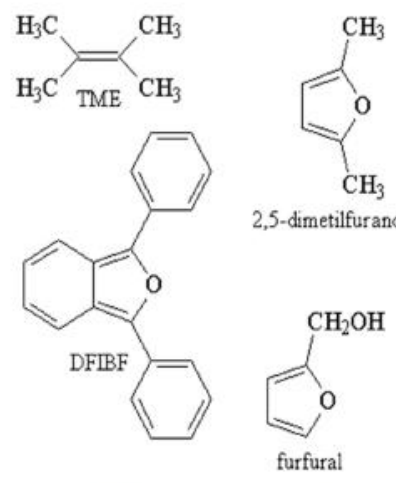

Obtención de $\Phi_{\Delta}$

Fig. 8 (a) Cuantificación de la producción de oxígeno singlete a través de un método de atrapamiento que hace uso de imidazol como atrapador. El endoperóxido formado reacciona con N,N-dimetil-pnitroso-anilina, un colorante amarillo detectable mediante espectrofotometría de absorción, dando lugar a productos de oxidación no coloreados. Por cada molécula de N,N-dimetil-p-nitroso-anilina que desaparece se requieren 12 moléculas de oxígeno singlete en promedio. (b) Diversos atrapadores usados para la cuantificación de oxígeno singlete: $T M E=$ tetrametiletileno; 2,5-dimetilfurano; $D F I B F=$ difenilisobenzofurano; furfural.

\section{REACTIVIDAD DEL OXÍGENO SINGLETE}

Como se citó anteriormente el oxígeno singlete es una especie con carácter electrófilo altamente reactiva que tiende a reaccionar con otras moléculas ricas en densidad electrónica.

En este sentido se han descrito las reacciones del oxígeno singlete indicadas en la Figura 9.

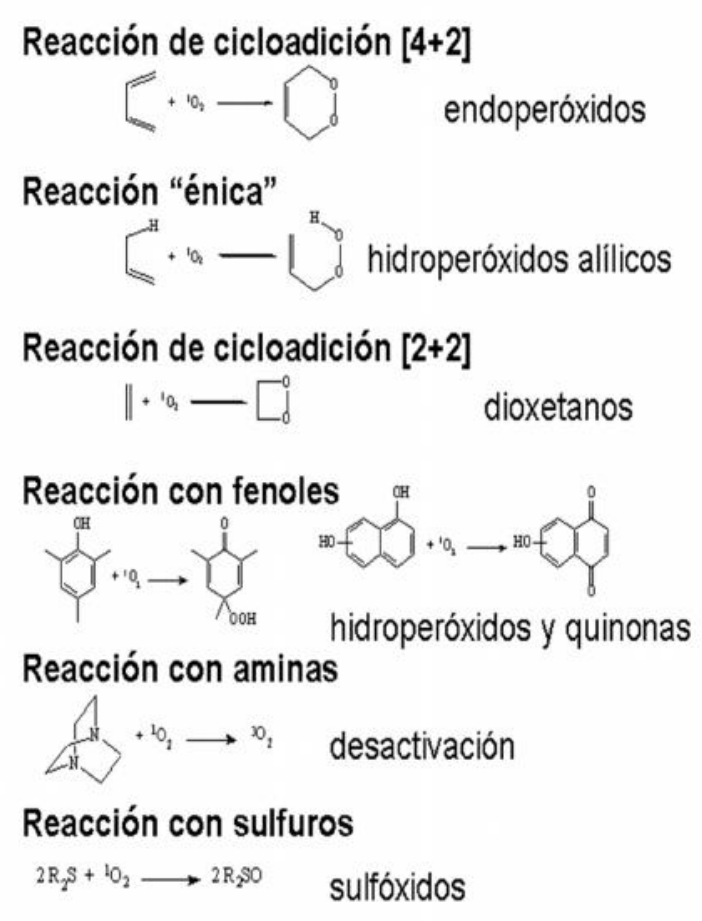

Fig. 9 Reacciones típicas del oxígeno singlete. 


\section{CONCLUSIONES}

Estas reacciones conducen normalmente a productos de oxidación por lo que, a parte de aplicaciones típicas en el campo de la síntesis en química orgánica, como por ejemplo la preparación del óxido de rosa (una esencia de alto valor añadido utilizada en perfumería) también se encuentran aplicaciones basadas en la degradación de contaminantes orgánicos a través de mecanismos de oxidación que, aunque no conduzcan a la mineralización total de las moléculas orgánicas, sí pueden dar lugar a especies oxidadas menos contaminantes. Finalmente, entre las aplicaciones más recientes del oxígeno singlete se encuentra el desarrollo de procesos de desinfección de aguas ya que el oxígeno singlete es susceptible de reaccionar con biomoléculas presentes en la pared celular de microorganismos, como son los lípidos y proteínas de membrana, lo que conduciría a su inactivación $\mathrm{y}$, por tanto, a la desinfección del medio acuoso en el que se encuentren presentes. Este tema será el objeto de otra de las presentaciones que se van a exponer a lo largo de este simposio.

\section{REFERENCIAS}

1. Wilkinson, F., Helman, W. P., Ross, A. B., “J. Phys. Chem". Ref. Data 1993, pp. 22, 113.
2. Braun, M., Maurette, M. T., Oliveros, E., "Photochemical Technology", Wiley, Chichester, 1991.

3. Turro, N. J., "Modern Molecular Photochemistry", University Science Books, Mill Valley, CA, 1991.

4. Baggott, G. J., "Essentials of Molecular Photochemistry", Blackwell, Oxford, 1991.

5. Kagan, J., "Organic Photochemistry. Principles and Applications", Academic, NY, 1997.

6. D. García-Fresnadillo., Georgiadou, Y., Orellana, G., Braun, A., M., Oliveros, E., "Helv. Chim. Acta", 1996, pp.79, 1222.

7. Gutiérrez, M. I., Martínez, C. G., D. García-Fresnadillo, Castro, A. M., Orellana, G., Braun, A. M., Oliveros, E., “J. Phys. Chem. A", 2003, 107, 3397.

8. http://qf.iqs.url.es/singlet_oxygen/

9. http://www.rcdc.nd.edu/compilations/SingOx/ SingOx.htm, y también http://www.rcdc.nd.edu/compilations/qy/QY. pdf

10. http://www.photobiology.info/

11._dgfresna@quim.ucm.es

Correspondencia: dgfresna@quim.ucm.es 